\title{
Obstruction of extrahepatic bile ducts by lymphocytes is regulated by IFN- $\gamma$ in experimental biliary atresia
}

\author{
Pranavkumar Shivakumar, ${ }^{1}$ Kathleen M. Campbell, ${ }^{1}$ Gregg E. Sabla, ${ }^{1}$ Alexander Miethke, ${ }^{1}$ \\ Greg Tiao,, ${ }^{2}$ Monica M. McNeal,, ${ }^{1}$ Richard L. Ward, ${ }^{1}$ and Jorge A. Bezerra ${ }^{1}$ \\ 'Department of Pediatrics and 2Department of Surgery, Cincinnati Children's Hospital Medical Center \\ and the University of Cincinnati College of Medicine, Cincinnati, Ohio, USA.
}

\begin{abstract}
The etiology and pathogenesis of bile duct obstruction in children with biliary atresia are largely unknown. We have previously reported that, despite phenotypic heterogeneity, genomic signatures of livers from patients display a proinflammatory phenotype. Here, we address the hypothesis that production of IFN- $\gamma$ is a key pathogenic mechanism of disease using a mouse model of rotavirus-induced biliary atresia. We found that rotavirus infection of neonatal mice has a unique tropism to bile duct cells, and it triggers a hepatobiliary inflammation by IFN- $\gamma$-producing $\mathrm{CD}^{+}$and $\mathrm{CD8}^{+}$lymphocytes. The inflammation is tissue specific, resulting in progressive jaundice, growth failure, and greater than $90 \%$ mortality due to obstruction of extrahepatic bile ducts. In this model, the genetic loss of IFN- $\gamma$ did not alter the onset of jaundice, but it remarkably suppressed the tissue-specific targeting of $T$ lymphocytes and completely prevented the inflammatory and fibrosing obstruction of extrahepatic bile ducts. As a consequence, jaundice resolved, and long-term survival improved to greater than $80 \%$. Notably, administration of recombinant IFN- $\gamma$ led to recurrence of bile duct obstruction following rotavirus infection of IFN- $\gamma$-deficient mice. Thus, IFN- $\gamma$-driven obstruction of bile ducts is a key pathogenic mechanism of disease and may constitute a therapeutic target to block disease progression in patients with biliary atresia.
\end{abstract}

\section{Introduction}

Biliary atresia is the most common cause of chronic liver disease in children and the prime indication for pediatric liver transplantation worldwide (1). The disease begins in the first few weeks of life with impaired bile flow due to the progressive obliteration of extrahepatic bile ducts; the onset of jaundice, acholic stools, and hepatosplenomegaly in otherwise healthy looking neonates are hallmarks of the disease. Although surgical creation of a biliary conduit has the potential to improve bile drainage, ongoing cholestasis and cirrhosis are frequent outcomes, at which time liver transplantation is the only hope for long-term survival. The development of nontransplant therapeutic modalities for children with biliary atresia has been hampered by limited knowledge of etiology and presumed multifactorial pathogenesis of disease (1-3). Despite phenotypic heterogeneity, the development of disease in the early postnatal period and the inflammatory injury of extrahepatic bile ducts occur uniformly in all patients and are consistent with a common biological process. Using liver biopsy samples from infants at different stages of disease, we found unique genomic signatures of differential lymphocyte function at the time of diagnosis (4). The signatures displayed the overexpression of IFN- $\gamma$ and other $\mathrm{T}$ lymphocyte-enriched genes, even when inflammatory infiltrates were similar to diseased controls, implying differential activation states of similar cell types.

Nonstandard abbreviations used: cytokeratin-7 (CK7); fluorescence-forming units (ffu); phycoerythrin (PE); rhesus rotavirus (RRV).

Conflict of interest: The authors have declared that no conflict of interest exists.

Citation for this article: J. Clin. Invest. 114:322-329 (2004)

doi:10.1172/JCI200421153
Direct proof of a cause and effect relationship between a Th1like, proinflammatory circuit and the hepatobiliary injury has met remarkable experimental challenges as a result of the inability to study human samples prior to or at the time of bile duct obstruction and the obvious ethical barriers to obtain normative data from livers of normal age-matched infants. Therefore, we used a mouse model of biliary atresia to test the hypothesis that IFN- $\gamma$ plays a key regulatory role in the pathogenesis of bile duct injury and obstruction in biliary atresia. We found that hepatic lymphocytes undergo Th1 commitment at the time of biliary injury and obstruction. More notably, loss of IFN- $\gamma$ expression completely prevented the inflammatory and fibrosing obstruction of bile ducts, which resulted in resolution of symptoms and improved long-term survival.

\section{Results}

Rotavirus infection in the immediate neonatal period results in bile duct obstruction. Infection of WT Balb/c mice with rhesus rotavirus (RRV) in the first 24 hours of life results in biliary obstruction and recapitulates the dramatic phenotype of biliary atresia, with generalized jaundice, acholic stools, and bilirubinuria developing in the neonatal period $(5,6)$. To identify dominant biological processes in this model, we inoculated $1.5 \times 10^{6}$ fluorescence-forming units (ffu) of RRV intraperitoneally into $25 \mathrm{WT}$ Balb/c mice within 24 hours of birth. Jaundice developed in approximately $80 \%$ of mice by 7 days of age, at which time the livers displayed expansion of portal triads by lymphocytic infiltrates and bile duct proliferation; the extrahepatic bile ducts appeared atretic, with lumenal obstruction by inflammatory cells (Figure 1, A-D), which was present in more than $90 \%$ of mice with jaundice. Focal stenosis of the common bile duct with variable degrees of proximal or distal cystic dila- 

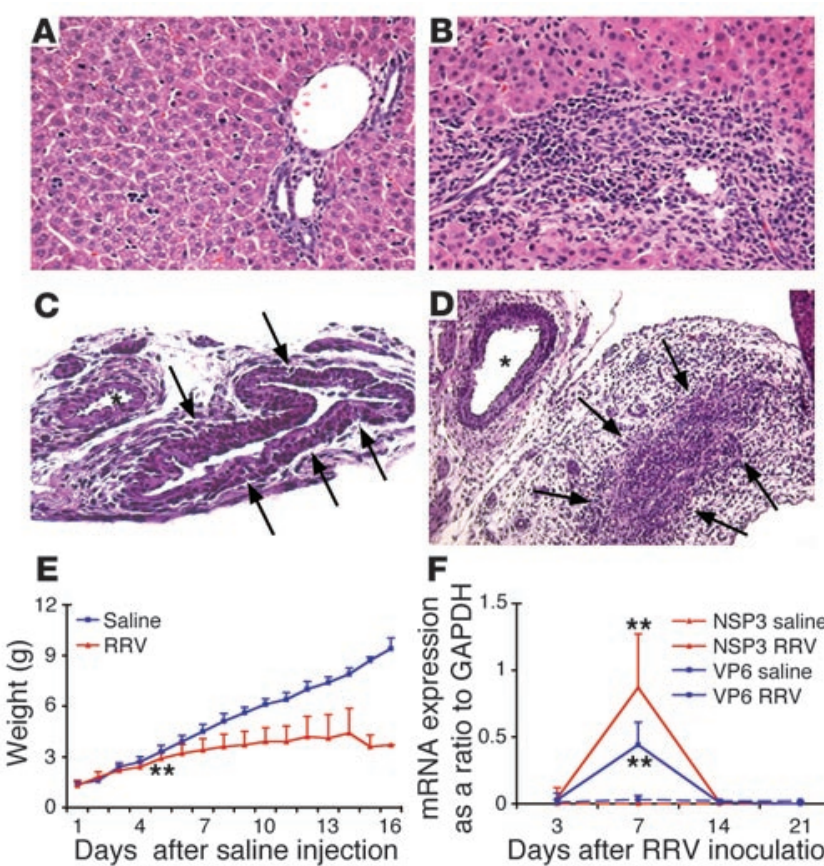

$\mathbf{F}$

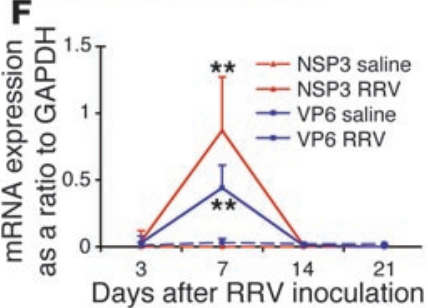

tation were also found in approximately $10 \%$ of symptomatic mice (data not shown). Following onset of symptoms, mice grew poorly, developed progressive cholestasis, and died between 14 and 21 days of age (Figure 1E). Despite the progressive cholestasis, mice were able to clear the virus from the hepatobiliary system, as demonstrated by their showing no detectable expression of mRNA encoding viral proteins (Figure 1F) or live virus (Supplemental Table 1; supplemental material available at http:/www.jci.org/cgi/content/ full/114/3/322/DC1) by 14 days after inoculation.

Rotavirus has tropism to cholangiocytes. The onset of cholestasis, effective viral clearance, and occurrence of minimal to no diarrhea in infected pups were in direct contrast to the reported diarrheal disease and absence of biliary symptoms when the pups are inoculated with RRV between 5 and 7 days of age (7). To determine the basis of this unexpected time-restricted biliary tropism, we determined the cellular target of RRV in the hepatobiliary system. Dual staining for RRV (Figure 2A) and cytokeratin-7 (CK7) (filament present in cholangiocytes; Figure $2 \mathrm{~B}$ ) identified RRV within the epithelium of extrahepatic bile ducts of infected mice (Figure 2C). A preferential staining of biliary epithelial cells was also supported by the dual immunostaining of these cells in the portal tract of liver sections from RRV-infected mice, with no staining of hepatocytes or other lobular cells (data not shown). In vitro, the infectious titer of RRV, as measured by a focus assay, is 100 -fold lower in a murine cholangiocyte line than in MA104 cells (monkey kidney epithelial cells known to be susceptible to RRV; see ref. 7). However, after infection of the cholangiocytes, recovery of

\section{Figure 1}

RRV infection induces biliary inflammation and growth failure in neonatal mice. WT Balb/c mice were injected with normal saline (control) or RRV within 24 hours of birth, and the hepatobiliary system was examined 7 days later. (A) While livers of control mice had normal appearance of the portal tracts, RRV challenge resulted in the expansion of portal spaces by inflammatory cells and proliferating bile duct cells (B). (C) Cross section of the extrahepatic bile duct of a control mouse revealed normal epithelium and unobstructed lumen (arrows). (D) In contrast, injection of RRV produced lumenal obstruction of extrahepatic bile ducts (arrows). Tissue sections were stained with H\&E. Magnification of $\times 400$ for A and B, $\times 200$ for C and D. Single asterisks denote neighboring arteries in $\mathbf{C}$ and $\mathbf{D}$. (E) It can be seen that RRV injection also led to poor growth during the suckling period. ${ }^{* *} P<0.01$ when compared with controls at days $7-16 ; n=25$ mice in the beginning of the experiment. Expression of mRNA encoding RRV nonstructural (NSP3) and structural (VP6) proteins was high at day 7 but $(\mathbf{F})$ undetectable at day $14 .{ }^{* *} P<0.01 ; n=4-7$ mice per group at each time point.

live virus is only approximately 11 -fold lower than in MA104 cells as measured in the MA104 cells (cholangiocytes, $0.6 \pm 0.4 \times 10^{8}$ $\mathrm{ffu} / \mathrm{ml}$ versus MA104 cells, $7.5 \pm 0.5 \times 10^{8} \mathrm{ffu} / \mathrm{ml}$ ). This suggests that the main difference lies in the decreased infectivity of RRV in cholangiocytes. When the same assays were applied to the murine hepatocyte line $\mathrm{H} 2.35$, recovery of live virus was only $2 \times 10^{4}-6 \times 10^{4}$ $\mathrm{ffu} / \mathrm{ml}$, again as determined in MA104 cells. Together, these data demonstrated that RRV had a unique tropism to the neonatal biliary epithelium. Although the full extent by which RRV modifies cholangiocyte survival is not yet known, the decreased susceptibility of cholangiocytes to RRV relative to MA104 cells in culture did not support a direct viral injury of cholangiocytes as the sole mechanism of injury to the epithelium and lumenal obstruction. Therefore, we investigated whether the inflammatory response to RRV played a regulatory role in tissue-specific injury.

IFN- $\gamma$-producing lymphocytes target the hepatobiliary system after viral challenge. Activation of inflammatory cells is a well-known mechanism associated with recognition and injury of target cells following a viral infection. One week after RRV inoculation of neonatal mice, portal tracts were infiltrated with lymphocytes. Applying dual-stain flow-cytometric analysis of mononuclear cells isolated from livers and spleens at different time points after RRV challenge, we found no changes in the population of $\mathrm{CD}^{+}, \mathrm{CD}^{+}$, or $\mathrm{CD}^{+}$lymphocytes in spleens of RRV-infected mice when compared with age-matched, saline-injected controls (data not shown). In contrast, livers of RRV-infected mice displayed a 3-fold increase in $\mathrm{CD}^{+}$lymphocytes above controls at days 7 and 14 (Figure $3 \mathrm{~A}$ ), which consisted of both $\mathrm{CD} 4^{+}$and $\mathrm{CD} 8^{+}$cells (Figure $3, \mathrm{C}$ and D). Interestingly, the number of CD $19^{+}$lymphocytes decreased remarkably in livers following RRV challenge (Figure 3B). In addition to the tissue-specific population of T lymphocytes in RRVinfected mice, livers had a significant increase in mRNA expression

\section{Figure 2}

RRV targets the biliary epithelium in neonatal mice. Fluorescence immunostaining shows specific signal for RRV (red, A) and the cholangiocyte cytoskeletal filament CK7 (green, B) in the epithelium of extrahepatic bile ducts 3 days after RRV inoculation, with colocalization of RRV and CK7 (yellow, C).
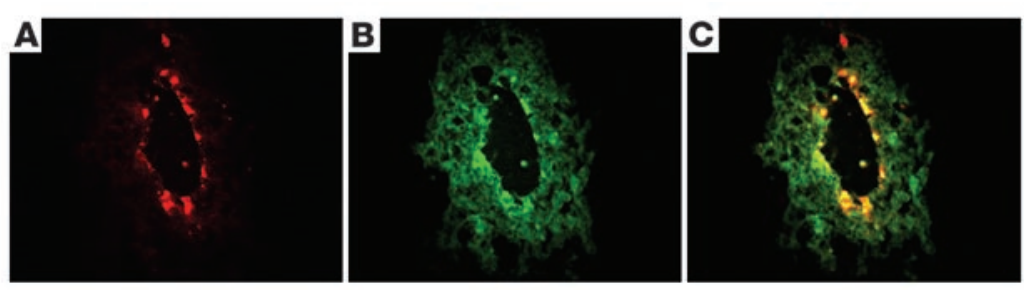

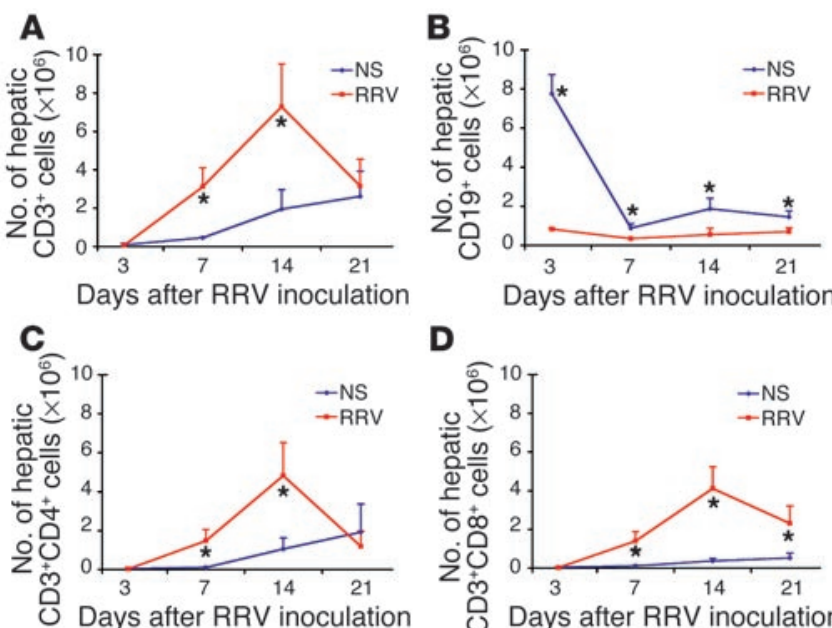

D

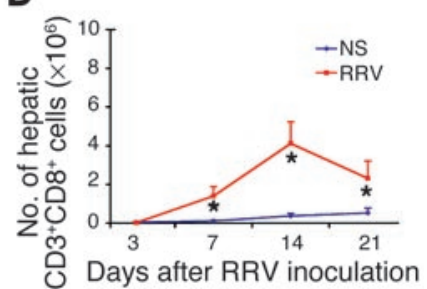

E

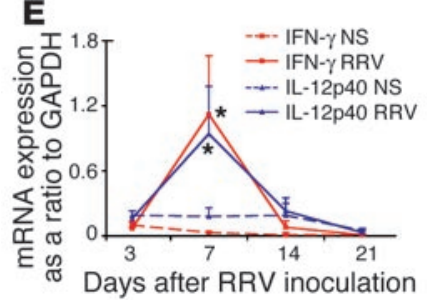

$\mathbf{F}$

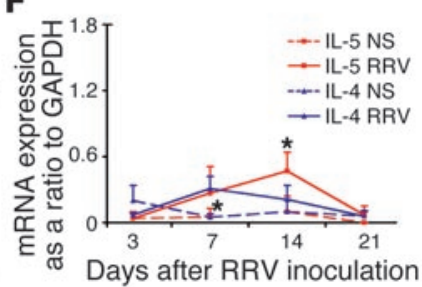

Figure 3

RRV infection results in Th1 polarization of hepatic lymphocytes. Hepatic cell surface staining by flow cytometry for CD3 (A) and CD19 (B) shows that the lymphocytic infiltrate in portal tracts is predominantly composed of $\mathrm{CD}^{+}$cells beginning 7 days after RRV inoculation. $\mathrm{CD}^{+}$ cells also showed staining with $\mathrm{CD}^{+}(\mathbf{C})$ or $\mathrm{CD}^{+}(\mathrm{D})$. (E) Functional polarization of $T$ lymphocytes is demonstrated 7 days after RRV challenge by an increase in mRNA expression for IFN- $\gamma$ and IL-12p40. (F) The mRNA expression for Th2 cytokines also increases above the levels of controls at 7 and 14 days, but at lower levels when compared with Th1 cytokines. ${ }^{*} P<0.05$ when the RRV group is compared with controls; $n=4-7$ mice per group at each time point. NS, normal saline.

of the Th1 cytokines IFN- $\gamma$ and IL-12p40, with a high level at 7 days and a return to baseline levels by 14 days (Figure $3 \mathrm{E}$ ), while the increase in the Th2 cytokines IL- 4 and IL-5 was milder and temporally delayed until 14 days for IL-5 (Figure 3F).

To determine the main inflammatory cells producing dominant Th1 cytokines, we used surface and intracellular labeling-based flow-cytometric analysis, and found that IFN- $\gamma$ expression increased more than 40 -fold in $\mathrm{CD}^{+} \mathrm{CD} 4^{+}$lymphocytes and approximately 18 -fold in $\mathrm{CD}^{+} \mathrm{CD}^{+}$lymphocytes at 7 days (Supplemental Figure $1, \mathrm{~A}$ and $\mathrm{B}$ ). The expression of $\mathrm{IL}-5$ by $\mathrm{CD}^{+}, \mathrm{CD}^{+}$, and $\mathrm{CD} 8^{+}$cells was low in RRV-infected livers (with highest levels of only 2- to 4.5fold at 7 and 14 days [Supplemental Figure 1, C and D]). These data demonstrated a striking temporospatial infiltration of the hepatobiliary system by $\mathrm{T}$ lymphocytes, with polarization to a Th1 phenotype at the onset of symptoms in infected mice.

Loss of IFN- $\gamma$ prevents bile duct obstruction and promotes long-term survival. IFN- $\gamma$ is an important effector of the Th1 phenotype and has been implicated in the pathogenesis of autoimmune disorders (8). In this context, the overexpression of IFN- $\gamma$ in livers of RRVinoculated mice and of infants with biliary atresia was consistent with a regulatory role of IFN- $\gamma$ in the inflammatory injury and obstruction of the bile ducts in biliary atresia. To directly test this claim, we inoculated mice carrying the genetic inactivation of the IFN- $\gamma$ gene $\left(I F N-\gamma^{-/-}\right)$and WT Balb/c mice (9) with RRV. $\mathrm{RRV}$ infection resulted in persistent cholestasis in approximately $80 \%$ of WT Balb/c mice (Figure 4), and fewer than 10\% survived beyond 21 days. In contrast, although jaundice, acholic stools, and bilirubinuria developed in $90-100 \%$ of $I F N-\gamma^{-/-}$mice 3-5 days after RRV challenge in a fashion similar to what occurred in WT Balb/c mice, acholic stools resolved in between 8 and 13 days; this was followed by a complete resolution of jaundice and bilirubinuria (Figure 4) and greater than $80 \%$ survival beyond 21 days.

The resolution of jaundice in the absence of IFN- $\gamma$ suggested that the extrahepatic ductal system was not obstructed. To determine the anatomical basis of jaundice clearance, we examined livers and extrahepatic bile ducts of WT Balb/c and $I F N-\gamma^{-/-}$mice challenged with RRV ( $n=15-20$ mice in each group). The hilum of WT Balb/c mice showed typically small, contracted gallbladders and atretic bile ducts (Figure 5, A and C); in stark contrast, gallbladders of IFN- $\gamma^{-/-}$mice contained bile, and bile ducts were unobstructed and maintained lumenal continuity with the duodenum (Figure 5, B and D). Histologically, extrahepatic bile ducts were obstructed by inflammatory cells (at 7 days) and extracellular matrix (at 14 days) in WT Balb/c mice (Figure 5, E and G), while the lumen of ducts of $I F N-\gamma^{-/-}$mice remained unobstructed (Figure 5, F and H). To further demonstrate the key role of IFN- $\gamma$ in duct obstruction, we administered recombinant IFN- $\gamma$ intraperitoneally to $18 \mathrm{IFN}-\gamma^{-/}$ mice every day after RRV inoculation until 14 days (time of killing) and examined the development of symptoms and obstruction of extrahepatic bile ducts. Restoration of the IFN- $\gamma$-sufficient state resulted in the timely development of cholestasis in more than $80 \%$ of the mice challenged with RRV, and the extrahepatic bile duct displayed recurrence of the obstruction in a fashion indistinguishable from the duct injury observed in WT Balb/c mice (Figure 6).

Detailed microscopic surveys of livers of both groups revealed lobular and portal inflammation with periductal infiltration by neutrophils at 3 days (Figure 7, A and B). Thereafter, these inflammatory changes were associated with portal expansion due to bile duct proliferation and inflammation at 7 days (Figure 7, C-F), which suggested that portal inflammation was an important influence in the development of cholestasis in infected mice of both genotypes. Taken together, these findings demonstrated that the onset of cholestasis in IFN- $\gamma^{-/-}$mice resulted from intrahepatic inflammation and cholangitis after RRV challenge. While this inflammatory
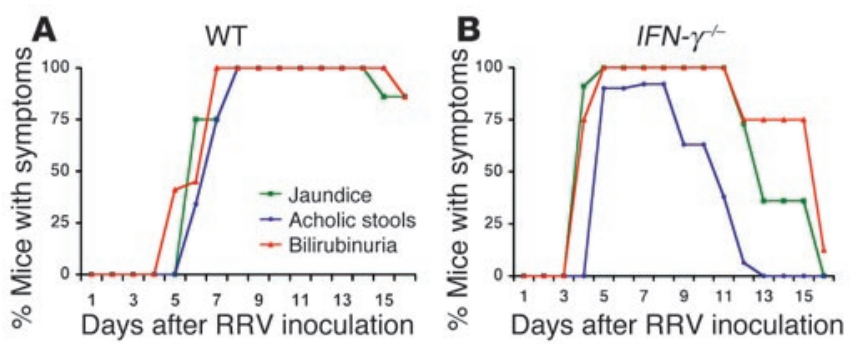

\section{Figure 4}

Loss of IFN- $\gamma$ improves symptoms of biliary obstruction after RRV challenge. Inoculation of RRV into newborn WT (A) mice induced jaundice, acholic stools, and bilirubinuria in all mice by 7 days, which persisted for the duration of the study in approximately $80 \%$ of mice. Although these symptoms also developed in IFN- $\gamma^{-1-}$ mice in a timely fashion (B), jaundice, acholic stools, and bilirubinuria resolved between 8 and 13 days after RRV challenge. $n=17$ for WT mice; $n=12$ for $I F N-\gamma^{-1}$ mice. 

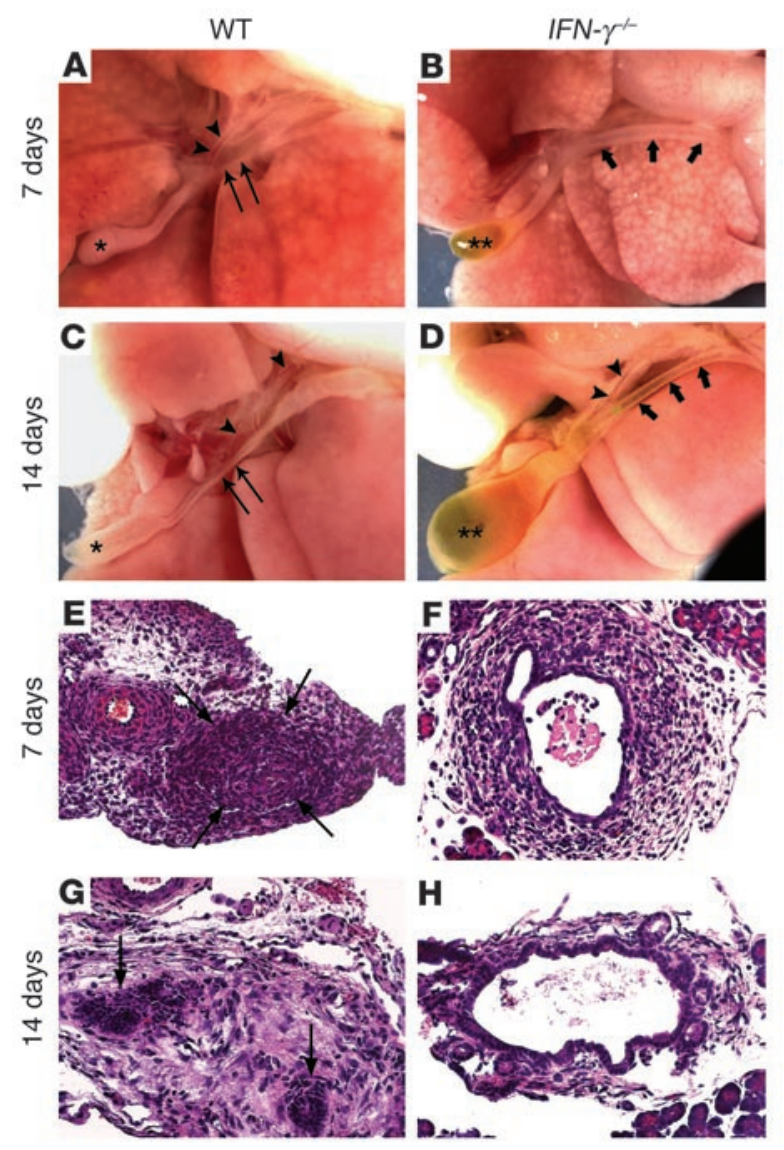

response progressed to complete ductal obstruction in WT Balb/c mice, loss of IFN- $\gamma$ prevented the excessive temporospatial accumulation of lymphocytes and obstruction of extrahepatic ducts.

IFN- $\gamma$ controls hepatic infiltration by $T$ lymphocytes. One notable histopathological difference between the portal inflammation of WT Balb/c and IFN- $\gamma^{-/-}$mice was that the inflammatory cells of $I F N-\gamma^{-/-}$mice consisted primarily of neutrophils at 7 days. The total number of neutrophils in a liver surface area of $6 \mathrm{~mm}^{2}$ at this time point increased in $I F N-\gamma^{-/-}$by $62 \%$ above that in WT Balb/c mice (mean \pm SD: $I F N-\gamma^{-/-}=146 \pm 41$ versus WT Balb/c $=91 \pm 28$; $P<0.05)$. The number of neutrophils at 14 days was similar in $I F N-\gamma^{-/-}$(mean \pm SD: $\left.36.6 \pm 13\right)$ and WT (mean \pm SD: $38 \pm 42$ ) mice; however, neutrophils composed the main inflammatory cell

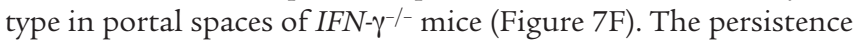
of a predominantly neutrophilic infiltration in the periductal microenvironment beyond the early phases of injury in IFN- $\gamma^{-/-}$mice pointed to a potential defect in the orderly succession of the cellular inflammatory response to biliary injury. To further define whether loss of IFN- $\gamma$ suppressed the population of livers by $\mathrm{T}$ lymphocytes, we quantified hepatic mononuclear cells by flow-cytometric analysis. We found a decrease of hepatic $\mathrm{CD}^{+}$lymphocytes following RRV challenge (Figure 8, A-C).

Despite the low number of $\mathrm{CD}^{+}$cells, loss of IFN- $\gamma$ did not impair the expression of IL-12p40, IL-4, and IL-5 in the liver (Figure 8 , D-F), did not change the tropism to biliary epithelium (Supplemental Figure 2), and did not impair the ability of the organism to clear RRV from the liver (Supplemental Figure 3). Because of previous reports describing the ability of IFN- $\alpha$ to prevent or attenuate

\section{Figure 5}

Loss of IFN- $\gamma$ prevents obstruction of extrahepatic bile ducts. Anatomical view of the hilum (A-D) of WT Balb/c mice displayed small, edematous gallbladders $\left({ }^{*}\right)$ at 7 and 14 days after RRV challenge, with long- (7 days) or short- (14 days) segment atresia of extrahepatic bile ducts (thin arrows). In contrast, IFN- $\gamma^{-1-}$ mice displayed gallbladders distended with bile $\left({ }^{*}\right)$ and unobstructed bile ducts (thick arrows). Arrowheads point to arterial vessels that follow extrahepatic bile ducts. Microscopically (E-H), bile ducts of WT Balb/c mice demonstrated lumenal obstruction by inflammatory cells (7 days) and extracellular matrix (14 days). In IFN- $\gamma^{-1-}$ mice, extrahepatic bile ducts had periductal inflammation and mild epithelial injury, but the lumen remained patent and without accumulation of matrix substrates at 7-14 days. Sections were stained with H\&E; magnification, $\times 200$; arrows in $(\mathbf{E})$ and $(\mathbf{G})$ denote obstructed bile ducts.

the development of biliary atresia when administered after RRV challenge $(10,11)$, we determined the hepatic mRNA expression of IFN- $\alpha$ and IFN- $\beta$ in WT Balb/c and IFN- $\gamma^{-/-}$mice after RRV infection. The levels of expression for IFN- $\alpha$ and IFN- $\beta$ increased in WT Balb/c mice at the onset of cholestasis, but the similar rise in $I F N-\gamma^{-/}$mice without development of biliary atresia did not support a direct link between IFN- $\alpha$ and bile duct obstruction (Supplemental Figure 4). Therefore, to explore the potential mechanisms by which IFN- $\gamma$ regulates the lymphocytic infiltration of the biliary system after RRV challenge, we determined the expression of Mig, IP-10, and I-Tac, three IFN- $\gamma$-regulated chemokines that control recruitment of activated lymphocytes to the liver (12-16). The expression of all three chemokines reached high levels at the onset of symptoms in WT Balb/c mice (7 days after RRV challenge). In IFN- $\gamma^{-/}$mice, the increase in hepatic expression was completely abolished for Mig and moderately suppressed for IP-10 and I-Tac (Figure 9). Collectively, these data demonstrated that IFN- $\gamma$ regulates the progression to biliary obstruction by $\mathrm{T}$ lymphocytes in response to an injury of cholangiocytes in the immediate postnatal period, possibly via key downstream chemotactic signals such as Mig.

\section{Discussion}

These data demonstrate that IFN- $\gamma$ plays a pivotal regulatory role in the obstruction of extrahepatic bile ducts in a mouse model of biliary atresia. In this model, the inflammatory and fibrosing obstruction of bile ducts temporally restricted to early postnatal development following RRV challenge results in progressive cholestasis and
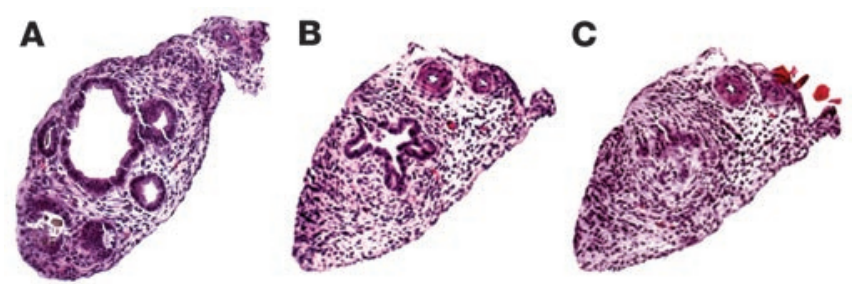

\section{Figure 6}

Administration of recombinant IFN- $\gamma$ results in obstruction of extrahepatic bile ducts in IFN- $\gamma^{-/}$mice. H\&E staining of transverse sections along the extrahepatic bile duct of an IFN- $\gamma^{-1-}$ mouse that received daily intraperitoneal injections of $2,000 \mathrm{U}$ of recombinant IFN- $\gamma$ per gram body weight following RRV challenge. (A) A patent proximal segment of the duct. (B) A narrow lumen with increasing periductal inflammation (approximately $700 \mu \mathrm{m}$ from the section in A). (C) Section approximately $100 \mu \mathrm{m}$ from the section in $\mathbf{B}$; the inflammation completely occludes the extrahepatic bile duct. Magnification $\times 200$. 

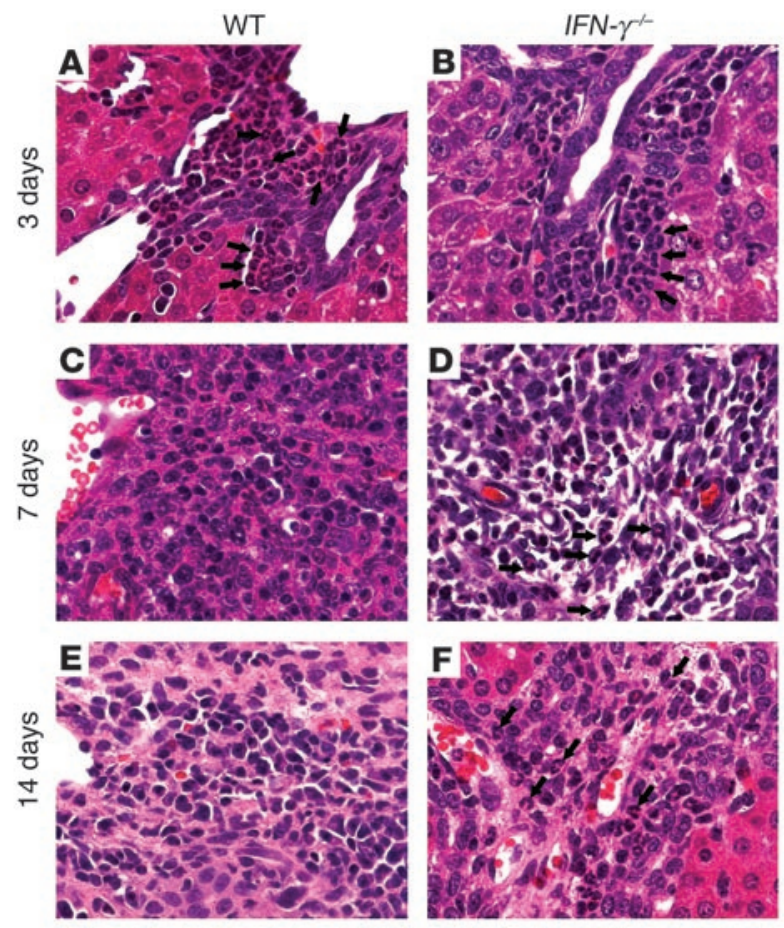

shares striking similarities with clinicopathological findings of biliary atresia in children. Although no viral agent has been consistently associated with biliary atresia in humans (1-3), the ability of RRV to target murine cholangiocytes and induce a tissue-specific inflammatory injury proves the principle that infectious agents may target neonatal cholangiocytes and trigger an undesired inflammatory response that results in occlusion of extrahepatic bile ducts. The hepatobiliary system of suckling mice has also been shown to be targeted by reovirus type 3 , but the inflammatory response has not been shown to cause obstruction of extrahepatic bile ducts (17-20). In contrast, RRV challenge triggers an immediate infiltration of the hepatobiliary system by neutrophils, followed by predominantly Th1-committed, IFN- $\gamma$-producing $\mathrm{T}$ lymphocytes at the time of obstruction of extrahepatic bile ducts.

The pivotal role of lymphocytes and IFN- $\gamma$ in duct obstruction became evident in mice lacking IFN- $\gamma$. Without IFN- $\gamma$, the sequential switch to a lymphocyte-based hepatic inflammation did not occur, duct obstruction was completely prevented, and extrahepatic bile ducts maintained lumenal continuity with the duodenum. In keeping with a central role for IFN- $\gamma$ in duct obstruction, administration of recombinant IFN- $\gamma$ following RRV infection resulted in recurrence of biliary atresia in IFN- $\gamma$-deficient mice. This outcome was in contrast to previous reports in which administration of IFN- $\alpha$ prevented the development of biliary obstruction following RRV infection of neonatal WT Balb/c mice

\section{Figure 8}

Hepatic population of T lymphocytes and cytokine expression in IFN- $\gamma^{-1}$ mice. Flow cytometry shows a decreased population in the livers of IFN $-\gamma^{-1-}$ mice by $\mathrm{CD} 3^{+} \mathrm{CD} 4^{+}$and $\mathrm{CD} 3^{+} \mathrm{CD} 8^{+}$lymphocytes after RRV challenge (A-C). Despite the loss of IFN- $\gamma$, expression of hepatic mRNA expression for IL-2p40, IL-4, and IL-5 in response to RRV challenge does not change (D-F). $n=4-7$ mice per group at each time point. ${ }^{*} P<0.04$.

\section{Figure 7}

Persistent infiltration of portal space by neutrophils in IFN- $\gamma^{-1-}$ mice. RRV challenge induces a neutrophil-based pericholangitis within 3 days in WT and IFN- $\gamma^{-1-}$ mice (A and B). In WT mice, inflammatory cells switch to lymphocytes in expanded portal spaces 7-14 days after challenge (C and E). This switch is incomplete in IFN- $\gamma^{-1-}$ mice, which continue to display portal neutrophils ( $\mathbf{D}$ and $\mathbf{F}$ ). Arrows point to neutrophils.

$(11,12)$. Although these studies did not address the mechanisms used by IFN- $\alpha$ to prevent biliary obstruction, it is possible that IFN- $\alpha$ may interfere with early phases of biliary injury by a direct antiviral effect, by interfering with the interaction between RRV and cholangiocytes, or by blocking the inflammatory response to viral challenge. Our studies did not formally investigate how IFN- $\alpha$ regulates biliary obstruction following RRV challenge, but the findings of a similar increase in mRNA expression for both IFN- $\alpha$ and IFN- $\beta$ after RRV challenge in WT mice (with biliary obstruction) and IFN- $\gamma$-deficient mice (without biliary obstruction) do not support a direct regulatory role for IFN- $\alpha$ in duct obstruction. Collectively, these data provide direct evidence for a singular role of IFN- $\gamma$ in duct obstruction by lymphocytes in neonatal mice. In light of the shared phenotypic and molecular features between this model and children with biliary atresia (4), we propose that IFN- $\gamma$ may play a central role in pathogenic mechanisms of disease.

Our findings also provide strong evidence that the pathogenic mechanisms of biliary atresia conform to a biological continuum previously not recognized $(1,2,21)$. The initiating events of this continuum, namely the insult by a viral agent and the immediate neutrophil-based inflammatory response, were not affected by IFN- $\gamma$. In contrast, the progression to ductal obliteration by inflammatory cells was prevented by loss of IFN- $\gamma$, either through

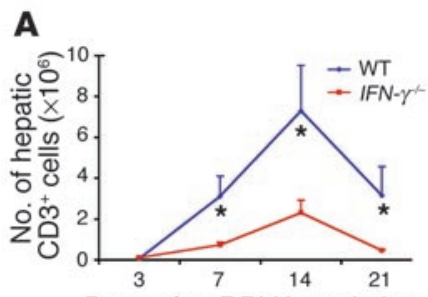

Days after RRV inoculation

B
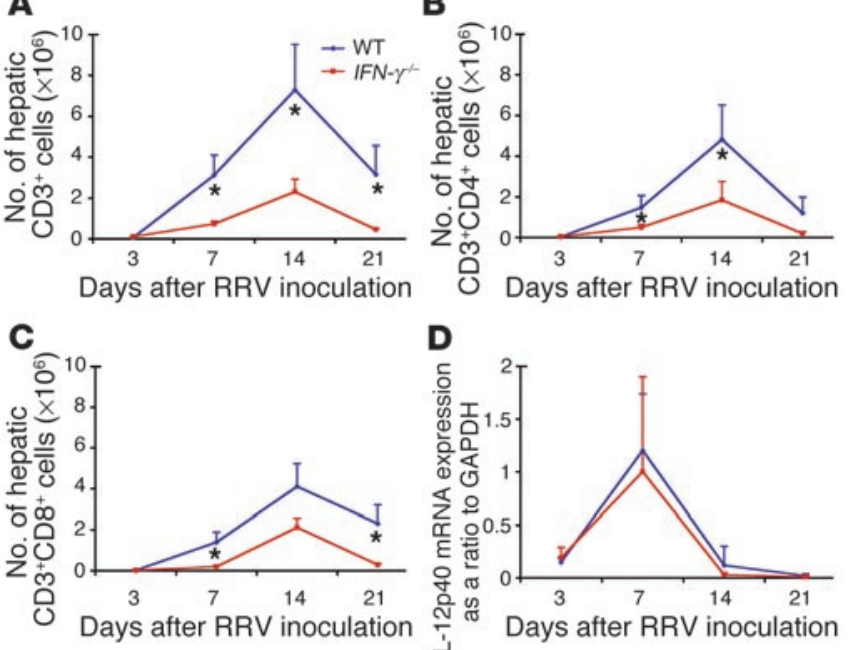

D
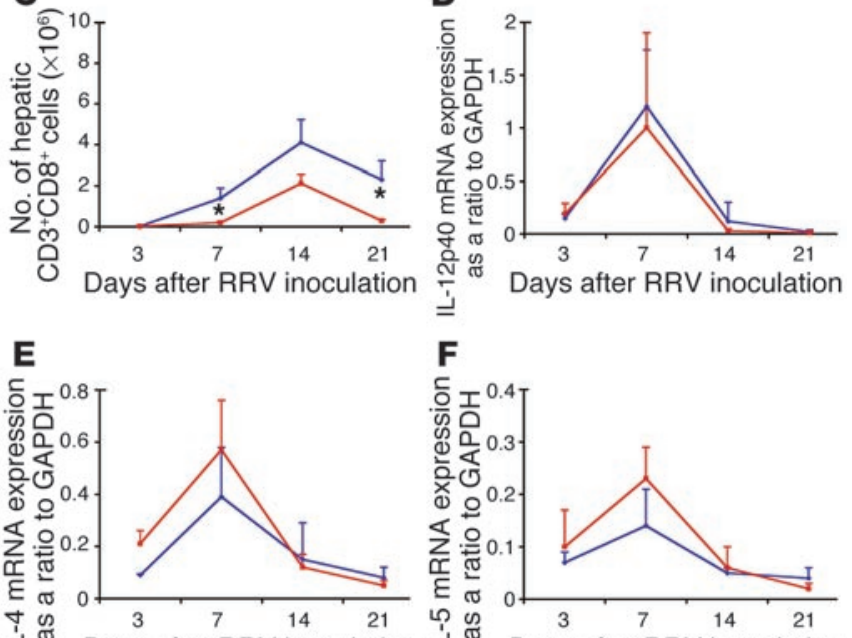

Days after RRV inoculation 


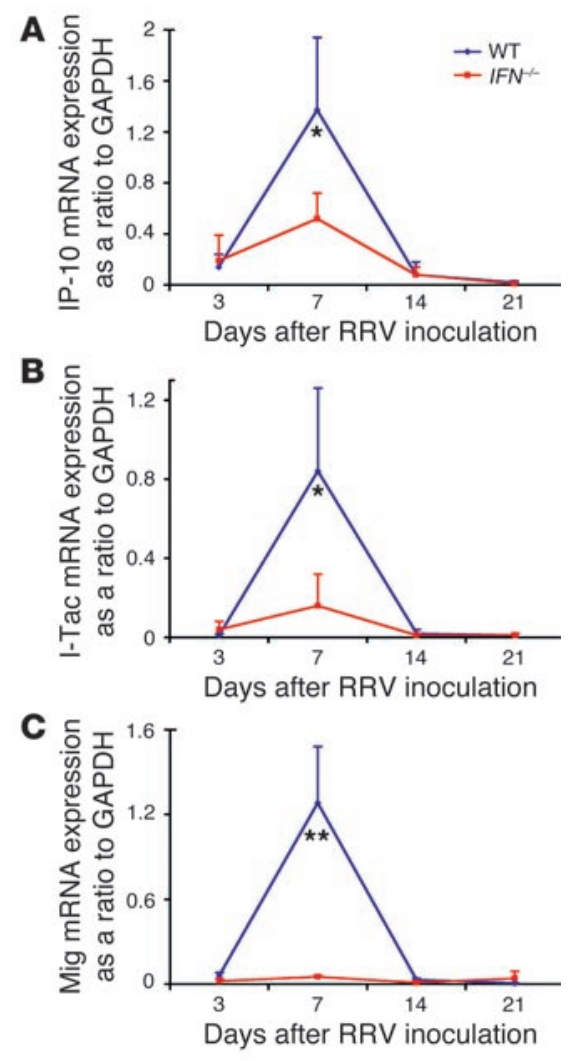

a direct interference with the tropism of $\mathrm{T}$ lymphocytes to the hepatobiliary system or indirectly through the suppression of downstream targets, such as $\operatorname{Mig}(15,16,22)$. The final step of ductal fibrosis, in which inflammatory cells are replaced by the concentric deposition of matrix substrates, was also prevented by loss of IFN- $\gamma$. On the basis of the antifibrogenic properties of IFN- $\gamma$ in the liver $(23,24)$, it is improbable that the lack of fibrosis is a direct consequence of the decrease in IFN- $\gamma$ production. Instead, lack of progression to ductal fibrosis probably represents an indirect consequence of the lack of intraductal inflammation induced by the loss of IFN- $\gamma$, which allows for restoration of epithelial integrity.

The argument for a biological continuum in the pathogenesis of biliary atresia does not rule out an important role of predisposing variables in disease susceptibility. The existence of such variables is supported by the variable degrees of biliary injury induced by RRV in different mouse strains (12). Furthermore, animal- and patientbased studies implicate specific genes in the pathogenesis of a subgroup of patients with biliary atresia who also display defects in laterality $(2,25-27)$. The presence of mutations in laterality genes in patients with extrahepatic malformations, but not consistently in patients who also have biliary atresia $(27,28)$, points to a modifying but not causative role for these genes in disease pathogenesis. Here, we began dissecting the molecular pathways regulating the pathogenic mechanisms responsible for the common inflammatory injury of the biliary system and identified IFN- $\gamma$ as a key regulator of ductal obstruction during the progression of disease. Logical followup studies will be crucial to determine whether IFN- $\gamma$ works in the biliary microenvironment by promoting lymphocyte survival and recruitment and/or by inducing epithelial growth to restore the epithelium of injured ducts, perhaps through downstream molecular

\section{Figure 9}

Impaired expression of chemokines following RRV challenge of IFN- $\gamma^{-1-}$ mice. Hepatic mRNA expression for downstream targets of IFN- $\gamma$ as a ratio to GAPDH shows a lower increase for IP-10 (A) and I-Tac (B) 7 days after RRV challenge, and a complete suppression of Mig (C). ${ }^{*} P<0.05$ and ${ }^{* *} P<0.001$ when WT is compared to IFN $-\gamma^{-1-}$ mice; $n=4-7$ mice for each group and at all time points.

targets such as the CXCR3 ligands Mig, IP-10, and I-Tac (29).

In summary, we used a mouse model of biliary atresia to explore the claim from human-based studies that expression of IFN- $\gamma$ may be an important pathogenic mechanism of disease (4). Notably, the absence of IFN- $\gamma$ expression in vivo produced no obvious interference with the host's susceptibility to bile duct injury following viral challenge or with the initial response orchestrating an acute inflammatory cholangitis. As a consequence, jaundice and acholic stools developed in a timely fashion, but the surprising resolution of symptoms implied patency of the biliary system. Within the liver, IFN- $\gamma$ deficiency minimized the expansion of periductal inflammation by $\mathrm{T}$ lymphocytes at later phases of injury. More notably, extrahepatic bile ducts displayed orderly lining by epithelial cells and were free of obstruction, maintaining lumenal continuity and bile flow between the liver and the duodenum. These data do not necessarily prove that IFN- $\gamma$ regulates biliary obstruction in humans; however, the remarkable similarities in onset of disease restricted to early postnatal stage (30), histopathological features $(5,31)$, and molecular signatures toward proinflammatory cytokines shared by this murine model and infants with biliary atresia underscore shared pathogenic mechanisms of disease. Therefore, new patient-based studies addressing genetic heterogeneity for IFN- $\gamma$ and counter-regulatory cytokines will be logical steps to validate a functional role of IFN- $\gamma$ in disease pathogenesis in humans. In this context, the identification of IFN- $\gamma$ will have broad implications for the development of novel therapeutic targets to block progression of disease and foster long-term survival with the native liver in children with biliary atresia.

\section{Methods}

Infection of neonatal mice with $R R V$. Balb/c mice were maintained in a specific pathogen-free vivarium and housed in a room with a 12-hour dark-light cycle. WT Balb/c and $I F N-\gamma^{-/-}$mice were injected with $0.9 \%$ saline solution (controls) or $1.5 \times 10^{6} \mathrm{ffu}$ of RRV intraperitoneally within 24 hours of birth. The strain of RRV used was generously provided by Marie Riepenhoff-Talty (University at Buffalo, The State University of New York, Buffalo, New York, USA) (5) and titered in MA104 cells by a fluorescence focus assay prior to injection as described previously (32). Infected mice that died within the first 2 days or that were not fed by their mothers after infection were excluded from further analysis. All mice were weighed daily and examined for the development of icterus of the skin not covered with fur, of acholic stools, and of bilirubinuria (using Multistix 10SG strips; Bayer Corp., Elkhart, Indiana, USA). Mice were killed at 3, 7, 14, and 21 days after saline or RRV injection. At these time points, gross appearance of livers and bile ducts was recorded, and organs were harvested for RNA isolation and histological analyses; whole livers were isolated from separate groups of mice and used for isolation of mononuclear cells. The Institutional Animal Care and Use Committee of the Children's Hospital Research Foundation (Cincinnati, Ohio, USA) approved all animal protocols.

Studies in IFN- $\gamma^{-/-}$mice. A colony of IFN- $\gamma^{-/-}$mice in the Balb/c strain was generated by backcrossing for more than 10 generations and donated to these studies by Elizabeth Majane, project officer of the NIAID/Taconic 
Repository (Taconic, Germantown, New York, USA). These mice were subjected to infection with RRV as just described. To determine the regulatory role of IFN- $\gamma$ in biliary obstruction, we administered once-daily intraperitoneal injections of 2,000 units of recombinant IFN- $\gamma$ per gram of body weight into IFN- $\gamma^{-1-}$ mice for the first 14 days after RRV inoculation (IFN- $\gamma=1 \times 10^{7}$ units/mg; Peprotech Inc., Rocky Hill, New Jersey, USA). This dose has been shown to efficiently restore the IFN- $\gamma$-sufficient state without obvious adverse systemic consequences in neonatal mice (33-35).

Histopathology and immunofluorescence. Livers and extrahepatic bile ducts were harvested from neonatal mice using a dissecting microscope. Tissues were paraffin-embedded, sectioned, and stained with H\&E for microscopic analysis. Sections were also used for histochemical localization of esterase activity (Leder staining) to identify neutrophils based on positive staining and morphology of nuclear segmentation as described previously (36). Stained cells were expressed as the total number of cells in a $6-\mathrm{mm}^{2}$ surface area of paraffin-embedded liver sections. For immunofluorescence, tissues were immersed in Histo Prep O.C.T. compound (Fisher Scientific Co., Fair Lawn, New Jersey, USA) and frozen on dry ice. Cryostat sections were fixed with cold acetone, nonspecific binding was blocked with M.O.M. mouse IgG blocking reagent (Vector Laboratories Inc., Burlingame, California, USA), and sections were incubated first with monoclonal anti-CK7 antibody (Accurate Chemical \& Scientific Corporation, Westbury, New York, USA) diluted 1:100, followed by rabbit anti-RRV antibody diluted 1:4,000 (32). Specific signals were detected using FITC-conjugated anti-mouse antibody and Texas Red-conjugated anti-rabbit antibody (both from Jackson ImmunoResearch Laboratories Inc., West Grove, Pennsylvania, USA), and a Zeiss Axiophot 2 microscope (Carl Zeiss Inc., Thornwood, New York, USA) using FITC and Rhodamine filters.

Susceptibility of cholangiocytes to $R R V$. Known titers of RRV were incubated with a murine cholangiocyte cell line isolated from WT Balb/c mice (here named mCL; ref. 37), murine hepatocytes H2.35 (ATCC no. CRL-1995), and embryonic African green monkey kidney epithelial cells MA104 (ATCC no. CRL-2378) in triplicate for 1 hour. Thereafter, cells were incubated with virus-free medium for 48 hours, and the infectious titer of RRV was determined by a focus assay as described previously (38).

Expression of mRNA encoding viral proteins and murine cytokines. Total RNA was extracted from different mouse tissues using TRIzol reagent (Life Technologies Inc., Carlsbad, California, USA), and integrity was confirmed by agarose gel electrophoresis as described previously (39). Following incubation with RNase-free DNase I (Life Technologies Inc.), reverse transcription was performed with SuperScript II reverse transcriptase and oligo(dT) $)_{12-18}$ (Life Technologies Inc.) according to the manufacturer's instructions. cDNA was subjected to real-time kinetic PCR on a Stratagene Mx-4000 Multiplex Quantitative PCR sequence detector (Stratagene, La Jolla, California, USA) using SYBR Green I as a double-strand DNA-specific binding dye to quantify expression for IFN- $\gamma$, IFN- $\alpha$, IFN- $\beta$, IL-12p40, IL-4, IL-5, Mig, IP-10, I-Tac, the RRV structural protein VP6, and the nonstructural protein NSP3. PCR amplifications were performed with specific primers (Supplemental Table 2) in a total volume of $20 \mu \mathrm{l}$ containing $0.1 \mathrm{pmol}$ of each primer, $10 \mu \mathrm{l}$ of $2 \times$ Brilliant SYBR Green QPCR Master Mix (Stratagene), $30 \mathrm{nM}$ of 1:500 diluted reference dye (ROX), 1 $\mu \mathrm{l}$ of 1:10 diluted cDNA and nuclease-free PCR-grade water, after initial denaturation at $95^{\circ} \mathrm{C}$ and $40-45$ cycles $\left(95^{\circ} \mathrm{C}\right.$ for 30 seconds, $55^{\circ} \mathrm{C}$-for 1 minute, and $72^{\circ} \mathrm{C}$ for 30 seconds).

Isolation and fluorometric analysis of cells from livers and spleens. Mononuclear cells were isolated from freshly harvested spleens and livers of RRV- or salineinjected mice by gentle homogenization of the tissue in RPMI Medium 1640 (Life Technologies Inc., Grand Island, New York, USA passage through a 40$\mu \mathrm{m}$ Cell Strainer (BD, Franklin Lakes, New Jersey, USA), and centrifugation at $270 \mathrm{~g}$ for 10 minutes at $4^{\circ} \mathrm{C}$. The supernatant was discarded, and cells were washed once with PBS containing 4\% FCS (Life Technologies Inc.) and recentrifuged. The cell pellet was resuspended in $10 \mathrm{ml}$ of RBC lysis buffer (0.15 $\mathrm{M} \mathrm{NH}_{4} \mathrm{Cl}, 10 \mathrm{mM} \mathrm{KHCO}_{3}$, and $0.1 \mathrm{mM} \mathrm{Na}_{2}$ EDTA at $\mathrm{pH}$ 7.2). After incubation for 5 minutes at $4{ }^{\circ} \mathrm{C}$, cells were harvested by centrifugation and washed once with PBS- $4 \%$ FCS before further analysis.

To perform two-color flow-cytometric analyses, mononuclear cells from the liver and spleen were resuspended in a concentration of $1 \times 10^{7}-1 \times 10^{8}$ cells $/ \mathrm{ml}$ in FACS buffer (PBS containing $0.1 \%$ [w/v] sodium azide and $2 \%$ [v/v] FCS) and added to a V-bottom, 96-well microtiter plate (Corning Inc., Corning, New York, USA) at a volume of $200 \mu \mathrm{l} /$ well. Cell staining for flow cytometry was conducted as described previously (40). In brief, cells were preincubated with 1:200 dilution of anti-mouse FcyII/III receptor mAb CD16/CD32 (2.4G2) for 15 minutes at $4{ }^{\circ} \mathrm{C}$ in the dark to block nonspecific adherence of $\mathrm{mAbs}$ to $\mathrm{Fc}$ receptors. The cells were surface stained by incubating with fluorochrome-conjugated $\mathrm{mAbs}$ at a concentration of 10 $\mu \mathrm{g} / \mathrm{ml}$, for 30 minutes at $4^{\circ} \mathrm{C}$ in the dark. The following antibodies were used: FITC- or phycoerythrin-conjugated (PE-conjugated) anti-mouse CD3 (17A2, IgG $\mathrm{G}_{2 \mathrm{~b}}$ ), anti-mouse CD4 (RM4-4, IgG $\mathrm{I}_{2 \mathrm{~b}}$ ), anti-mouse CD8a (53-6.7, $\operatorname{IgG}_{2 \mathrm{a}}$ ), and anti-mouse CD19 (1D3, $\operatorname{IgG}_{2 \mathrm{a}}$ ), all purchased from BD Biosciences (San Jose, California, USA). Background fluorescence was evaluated by staining the cells at optimal concentrations with isotype control antibodies. Cells were then analyzed using a FACSCalibur dual-laser flow cytometer (BD Biosciences), with excitation at 488 and $633 \mathrm{~nm}$. Data were analyzed using CellQuest software (BD Biosciences). For each sample, 20,000 events were analyzed.

Intracellular cytokine staining. Intracellular cytokine staining was performed using the Cytofix/Cytoperm (with GolgiPlug) kit according to the manufacturer's instruction (BD Biosciences). In brief, intrahepatic mononuclear cells were incubated with $1 \mu \mathrm{l} / \mathrm{ml}$ GolgiPlug (Brefeldin A) for 4 hours at $37^{\circ} \mathrm{C}$, followed by incubation with antibodies that recognize the FcyII/III receptors (2.4G2), FITC-conjugated anti-mouse CD4 (RM4-4, $\left.\mathrm{IgG}_{2 \mathrm{~b}}\right)$, and anti-mouse CD8a (53-6.7, $\operatorname{IgG}_{2 \mathrm{a}}$ ). The cells were washed twice with staining buffer, then fixed and permeabilized by incubating in $100 \mu \mathrm{l}$ of BD Cytofix/Cytoperm solution for 20 minutes at $4{ }^{\circ} \mathrm{C}$. Cells were then stained with PE-conjugated anti-IFN- $\gamma$ (XMG1.2, IgG I $_{1}$, anti-IL-5 (TRFK5, $\mathrm{IgG}_{1}$ ), or isotype control (PE-conjugated rat $\mathrm{IgG}_{1}$ ). Stained cells were analyzed using a FACSCalibur flow cytometer.

Statistical analysis. Values are expressed as mean $\pm \mathrm{SD}$, and statistical significance was determined by unpaired $t$ test, with a significance level of $P<0.05$.

\section{Acknowledgments}

This work was supported by grants from the Translational Research Initiative of the Cincinnati Children's Hospital Medical Center and NIH grants DK-64008 (to J.A. Bezerra) and DK-064403 (Digestive Disease Research Development Core). We thank William Balistreri and David Williams for insightful review of the manuscript; Bryan Donnelly and Steven Allen for assistance with viral assays; Marie Riepenhoff-Talty for donation of aliquots of RRV; Yoshi Ueno and James Boyer for donation of murine cholangiocytes; and Elizabeth Majane and the NIAID/Taconic Repository for providing $I F N-\gamma^{-/-}-\mathrm{Balb} / \mathrm{c}$ mice.

Received for publication January 26, 2004, and accepted in revised form June 8, 2004.

Address correspondence to: Jorge A. Bezerra, Division of Gastroenterology, Hepatology and Nutrition, Cincinnati Children's Hospital Medical Center, 3333 Burnet Avenue, Cincinnati, Ohio 452293039, USA. Phone: (513) 636-3008; Fax: (513) 636-5581. E-mail: jorge.bezerra@cchmc.org. 
1. Balistreri, W.F., et al. 1996. Biliary atresia: current concepts and research directions. Summary of a symposium. Hepatology. 23:1682-1692.

2. Perlmutter, D.H., and Shepherd, R.W. 2002. Extrahepatic biliary atresia: a disease or a phenotype? Hepatology. 35:1297-1304.

3. Sokol, R.J., Mack, C., Narkewicz, M.R., and Karrer, F.M. 2003. Pathogenesis and outcome of biliary atresia: current concepts. J. Pediatr. Gastroenterol. Nutr. 37:4-21.

4. Bezerra, J.A., et al. 2002. Genetic induction of proinflammatory immunity in children with biliary atresia. Lancet. 360:1563-1659.

5. Riepenhoff-Talty, M., et al. 1993. Group A rotaviruses produce extrahepatic biliary obstruction in orally inoculated newborn mice. Pediatr. Res. 33:394-399.

6. Petersen, C., et al. 1997. New aspects in a murine model for extrahepatic biliary atresia. J. Pediatr. Surg. 32:1190-1195.

7. Feng, N., Burns, J.W., Bracy, L., and Greenberg, H.B. 1994. Comparison of mucosal and systemic humoral immune responses and subsequent protection in mice orally inoculated with a homologous or a heterologous rotavirus. J. Virol. 68:7766-7773.

8. O'Shea, J.J., Ma, A., and Lipsky, P. 2002. Cytokines and autoimmunity. Nat. Rev. Immunol. 2:37-45.

9. Dalton, D.K., et al. 1993. Multiple defects of immune cell function in mice with disrupted interferon-gamma genes. Science. 259:1739-1742.

10. Petersen, C., Bruns, E., Kuske, M., and von Wussow, P. 1997. Treatment of extrahepatic biliary atresia with interferon-alpha in a murine infectious model. Pediatr. Res. 42:623-628.

11. Petersen, C., et al. 1998. Progress in developing animal models for biliary atresia. Eur. J. Pediatr. Surg. 8:137-141.

12. Cole, K.E., et al. 1998. Interferon-inducible T cell alpha chemoattractant (I-TAC): a novel non-ELR CXC chemokine with potent activity on activated $\mathrm{T}$ cells through selective high affinity binding to CXCR3. J. Exp. Med. 187:2009-2021.

13. Gasperini, S., et al. 1999. Gene expression and production of the monokine induced by IFN-gamma (MIG), IFN-inducible T cell alpha chemoattractant (I-TAC), and IFN-gamma-inducible protein-10 (IP-10) chemokines by human neutrophils. J. Immunol. 162:4928-4937.

14. Liu, M.T., Armstrong, D., Hamilton, T.A., and Lane, T.E. 2001. Expression of Mig (monokine induced by interferon-gamma) is important in T lymphocyte recruitment and host defense following viral infection of the central nervous system. J. Immunol. 166:1790-1795.

15. Shields, P.L., et al. 1999. Chemokine and chemokine receptor interactions provide a mechanism for selective $T$ cell recruitment to specific liver compartments within hepatitis $\mathrm{C}$-infected liver. J. Immunol. 163:6236-6243.

16. Arai, K., Liu, Z.X., Lane, T., and Dennert, G. 2002. IP-10 and Mig facilitate accumulation of T cells in the virus-infected liver. Cell Immunol. 219:48-56.

17. Barton, E.S., et al. 2003. Utilization of sialic acid as a coreceptor is required for reovirus-induced biliary disease. J. Clin. Invest. 111:1823-1833. doi: 10.1172/JCI200316303.

18. Szavay, P.O., Leonhardt, J., Czech-Schmidt, G., and Petersen, C. 2002. The role of reovirus type 3 infection in an established murine model for biliary atresia. Eur. J. Pediatr. Surg. 12:248-250.

19. Parashar, K., Tarlow, M.J., and McCrae, M.A. 1992. Experimental reovirus type 3 -induced murine biliary tract disease. J. Pediatr. Surg. 27:843-847.

20. Bangaru, B., Morecki, R., Glaser, J.H., Gartner, L.M., and Horwitz, M.S. 1980. Comparative studies of biliary atresia in the human newborn and reovirusinduced cholangitis in weanling mice. Lab. Invest. 43:456-462.

21. Sokol, R.J., and Mack, C. 2001. Etiopathogenesis of biliary atresia. Semin. Liver Dis. 21:517-524.

22. Kakimi, K., et al. 2001. Blocking chemokine responsive to gamma-2/interferon (IFN)-gamma inducible protein and monokine induced by IFN-gamma activity in vivo reduces the pathogenetic but not the antiviral potential of hepatitis B virus-specific cytotoxic T lymphocytes. J. Exp. Med. 194:1755-1766.

23. Baroni, G.S., et al. 1996. Interferon gamma decreases hepatic stellate cell activation and extracellular matrix deposition in rat liver fibrosis. Hepatology. 23:1189-1199.

24. Henri, S., et al. 2002. Cytokine regulation of periportal fibrosis in humans infected with Schistosoma mansoni: IFN-gamma is associated with protection against fibrosis and TNF-alpha with aggravation of disease. J. Immunol. 169:929-936.

25. Yokoyama, T., et al. 1993. Reversal of left-right asymmetry: a situs inversus mutation. Science. 260:679-682.

26. Mazziotti, M.V., et al. 1999. Anomalous development of the hepatobiliary system in the Inv mouse. Hepatology. 30:372-378.

27. Bamford, R.N., et al. 2000. Loss-of-function mutations in the EGF-CFC gene CFC1 are associated with human left-right laterality defects. Nat. Genet. 26:365-369.

28. Schon, P., et al. 2002. Identification, genomic organization, chromosomal mapping and mutation analysis of the human INV gene, the ortholog of a murine gene implicated in left-right axis development and biliary atresia. Hum. Genet. 110:157-165.

29. Koniaris, L.G., et al. 2001. Cytokine-responsive gene-2/IFN-inducible protein-10 expression in multiple models of liver and bile duct injury suggests a role in tissue regeneration. J. Immunol. 167:399-406

30. Czech-Schmidt, G., Verhagen, W., Szavay, P., Leonhardt, J., and Petersen, C. 2001. Immunological gap in the infectious animal model for biliary atresia. J. Surg. Res. 101:62-67.

31. Petersen, C., et al. 1997. New aspects in a murine model for extrahepatic biliary atresia. J. Pediatr. Surg. 32:1190-1195.

32. Estes, M.K., Graham, D.Y., Ramig, R.F., and Ericson, B.L. 1982. Heterogeneity in the structural glycoprotein (VP7) of simian rotavirus SA11. Virology. 122:8-14.

33. Donckier, V., et al. 1994. IFN-gamma prevents Th2 cell-mediated pathology after neonatal injection of semiallogeneic spleen cells in mice. J. Immunol. 153:2361-2368.

34. Hofstra, C.L., et al. 1998. Differential effects of endogenous and exogenous interferon-gamma on immunoglobulin E, cellular infiltration, and airway responsiveness in a murine model of allergic asthma. Am. J. Respir. Cell Mol. Biol. 19:826-835.

35. Schijns, V.E., Haagmans, B.L., and Horzinek, M.C. 1995 . IL-12 stimulates an antiviral type 1 cytokine response but lacks adjuvant activity in IFN-gamma-receptor-deficient mice. J. Immunol. 155:2525-2532.

36. Burstone, M.S. 1957. The cytochemical localization of esterase. J. Natl. Cancer Inst. 18:167-172.

37. Mano, Y., et al. 1998. Duct formation by immortalized mouse cholangiocytes: an in vitro model for cholangiopathies. Lab. Invest. 78:1467-1468.

38. Choi, A.H., McNeal, M.M., Basu, M., and Ward, R.L. 2000. Immunity to homologous rotavirus infection in adult mice: response. Trends Microbiol. 8:52.

39. Locaputo, S., Carrick, T.L., and Bezerra, J.A. 1999. Zonal regulation of gene expression during liver regeneration of urokinase transgenic mice. Hepatology. 29:1106-1113.

40. Salmon, M., et al. 1997. Inhibition of T cell apoptosis in the rheumatoid synovium. J. Clin. Invest. 99:439-446. 